Check for updates

The BMJ

Cite this as: BMJ 2021;373:n1543 http://dx.doi.org/10.1136/bmi.n1543 Published: 16 June 2021

\title{
Covid-19: Eight in 10 doctors have experienced moral distress during pandemic, BMA survey finds
}

\section{Abi Rimmer}

Nearly $80 \%$ of doctors say that the term "moral distress" resonates with their experiences of working during the pandemic, and over half say the same for "moral injury," a BMA survey has found.

The BMA ran a UK-wide survey on moral distress from 18 March to 12 April 2021 and received 1933 responses. Of 1864 respondents to a question on whether they had heard the term "moral distress" before, $56.2 \%$ of respondents (1048) said that they had, while 51.6\% (962) said that they had heard of "moral injury."

In its report on the survey findings, ${ }^{1}$ the BMA defined moral distress as "the psychological unease generated where professionals identify an ethically correct action to take but are constrained in their ability to take that action."

Moral injury, the BMA said, can arise where sustained moral distress leads to impaired function or longer term psychological harm. "Moral injury can produce profound guilt and shame, and in some cases also a sense of betrayal, anger and profound 'moral disorientation,"” it explained.

When doctors were presented with a definition, the survey found that most respondents said that the term "moral distress" resonated with their experiences at work $(78.4 \% ; 1409)$, and just over half $(51.1 \%$; 917) said that the term "moral injury" resonated with their experiences at work.

Doctors who worked with only covid-19 patients were more likely to say that moral distress $(88.9 \%$; 56$)$ or moral injury $(68.3 \%$; 43) resonated with their experiences than doctors who worked with non-covid patients (73.3\%; 332 (moral distress) and 45.4\%; 205 (moral injury)), the survey found.

Doctors from ethnic minority backgrounds (88.4\%; 343) were also more likely to report that moral distress resonated with their experiences at work than white doctors were $(75.6 \%$; 1017). Two thirds of doctors from ethnic minority backgrounds $(64.6 \% ; 250)$ also said that the term "moral injury" resonated with their experiences, compared with less than half of white doctors $(47.0 \% ; 631)$.

\section{Self-care plan}

Helen Fidler, deputy chair of the BMA's Consultants Committee, said that the results of the survey were incredibly worrying. "However, what is important is that being able to now name the struggles that many doctors are facing is the first step in helping to tackle these issues and pinpoint exactly what needs to be done to alleviate them," she said.

Fidler added that, like many other pre-existing pressures on staff in the NHS, the covid-19 pandemic had only exacerbated moral distress and moral injury.
"Perhaps moral distress and moral injury can never be eliminated, but it certainly shouldn't be a part of everyday life for our healthcare staff or their patients," she said. "All doctors want to do is to help others-it's why we're in this job-but when we're unable to make decisions that we believe are ethically right, it's not only distressing but also goes against every fibre of who we are: it's entrapping, it's stifling, and it directly impacts the care we give to our patients."

The BMA has called on the government to increase investment in the NHS, increase staffing levels, and provide emotional and psychological support for healthcare workers. It also said that doctors needed to be empowered and given clinical autonomy and that all workplaces should have an open and sharing culture.

The association also set out recommendations for doctors, which included talking about moral distress and moral injury, seeking advice where possible, and developing a self-care plan.

"Perhaps obvious, it is nevertheless important to reiterate the importance of medical professionals taking the time to look after themselves," the report said. "Physical and mental fatigue both contribute to moral distress and injury, potentially worsening their effects, and so finding the time for nutrition, exercise, and rest can all help."

BMA. Moral distress and moral injury: recognising and tackling it for UK doctors. Jun 2021. https://www.bma.org.uk/media/4209/bma-moral-distress-injury-survey-report-june-2021.pdf\#.

This article is made freely available for use in accordance with BMJ's website terms and conditions for the duration of the covid-19 pandemic or until otherwise determined by BMJ. You may use, download and print the article for any lawful, non-commercial purpose (including text and data mining) provided that all copyright notices and trade marks are retained. 